\title{
Low-frequency $\mathrm{H}$-reflex depression in trained human soleus after spinal cord injury
}

\author{
Richard K. Shields ${ }^{*}$, Shauna Dudley-Javoroski, and Preeti Deshpande Oza ${ }^{1}$ \\ Physical Therapy and Rehabilitation Science, The University of lowa, lowa City, IA, USA
}

\begin{abstract}
After spinal cord injury (SCI), widespread reorganization occurs within spinal reflex systems. Regular muscle activity may influence reorganization of spinal circuitry after SCI. The purpose of this study is to investigate the effects of long-term soleus training on H-reflex depression in humans after SCI. Seven subjects with acute ( $<7$ weeks) SCI (AC group) underwent testing of $\mathrm{H}-$ reflex depression at several frequencies of repetitive stimulation. Eight subjects (including 3 from AC) stimulated one soleus muscle daily, leaving the other leg as an untrained within-subject control. Trained limb H-reflexes were assessed during year 1 (TR1) and year 2 (TR2) of training. Untrained limbs were tested during year $2(\mathrm{UN})$. H-reflex amplitude was lower at 1,2 and $5 \mathrm{~Hz}$ than at 0.1 or $0.2 \mathrm{~Hz}(p<0.05)$. The pattern of depression differed between AC and $\mathrm{UN}$ ( $p<$ $0.05)$, but not between TR2 and UN $(p>0.05)$ despite significant adaptations in torque and fatigue resistance $(p<0.05)$. Three subjects who began training very early after SCI retained H-reflex post activation depression, suggesting that early intervention of daily muscular activity may be important.
\end{abstract}

\section{Keywords}

H-reflex; Fatigue; Torque; Electrical stimulation

\begin{abstract}
After spinal cord injury (SCI), extensive reorganization occurs in spinal reflex systems. Reorganization is often measured via the Hoffman reflex (H-reflex), a monosynaptic Ia afferent-mediated spinal reflex that normally exhibits frequency-dependent diminution of amplitude (low-frequency depression) with repetitive stimulation. After SCI in rats [21] and humans [17], H-reflexes cease to demonstrate low-frequency depression in response to repetitive stimulation. Lost supraspinal activation and subsequent reorganization of presynaptic inhibitory pathways may underlie this change and contribute to spasticity $[8,21]$.

In spinal transected rats, normal H-reflex depression can be recovered via introduction of passive exercise (cycling) [16]. Repeated activation of afferent pathways, particularly via Group I and II fibers may be critical for normalization of depression [14]. Human studies of individuals with incomplete SCI support that H-reflex excitability [10] and depression [15]
\end{abstract}

\footnotetext{
(C) 2011 Published by Elsevier Ireland Ltd.

*Corresponding author at: Physical Therapy and Rehabilitation Science, The University of Iowa, 1-252 Medical Education Building, Iowa City, IA 52242-1190, USA. Tel.: +1 319335 9791; fax: +1 319335 9707. richard-shields@ @iowa.edu (R.K. Shields).

${ }^{1}$ Present address: Physical Therapy Department, California State University, Fresno, CA, USA.
} 
are modifiable by volitional/assisted exercise (treadmill training, assisted stationary cycling). For subjects with complete SCI, the plasticity of the Hreflex in response to limb activity is unknown. Conditioning stimuli applied to the quadriceps had a highly variable effect on soleus H-reflex excitability in subjects with complete SCI [11]. Routine electrical stimulation of the paralyzed feline medial gastrocnemius yielded complete conversion to slow fibers with concurrent changes to type $\mathrm{S}$ motor units, supporting a periphery-to-motor neuron retrograde reorganization [13]. Several neurotrophic factors are known to be retrogradely transported from the periphery to the spinal cord [4]; up regulation of these factors after exercise $[6,7]$ suggests that muscle activity may increase the transport of these neuromodulatory factors to the spinal cord.

We previously observed that changes in the muscle periphery covaried in time with changes in H-reflex depression [17]. Bearing in mind recent discoveries regarding retrograde signaling mechanisms, we hypothesized that regular muscle activity of sufficient magnitude to arrest physiologic decline (torque, fatigue index) may be accompanied by normalization of associated spinal circuitry, as illustrated by H-reflex depression.

The purpose of this study is to investigate whether the soleus physiologic effects of training $[19,20]$ are accompanied by normalization of H-reflex depression in humans with complete SCI. We hypothesize that the trained limbs of subjects who perform 2 years of daily soleus stimulation will demonstrate $\mathrm{H}$-reflex depression during repetitive stimulation, but that their contralateral untrained limbs will show typical post-SCI loss of depression.

Twelve adults (two females) with complete motor and sensory SCI (ASIA-A) [2] participated in this study (Table 1). This protocol was approved by the University of Iowa's Human Subjects Office Institutional Review Board. All subjects provided written informed consent prior to participation. Exclusion criteria were lower motor neuron injury to the lumbar-sacral spinal segments, musculoskeletal injury to the paralyzed limbs, and history of fracture to the lower extremity. Two subjects (numbers 6 and 11) used anti-spasm medication throughout the study.

Seven subjects (the "Acute" cohort) underwent H-reflex testing of one limb before 7 weeks post-SCI elapsed. Three of these subjects (numbers 1, 5, and 6) then elected to enroll in the electrical stimulation training arm of the study. They along with five other subjects comprise the "Training" cohort.

Subjects in the Training group performed electrical stimulation of the right or left plantar flexor muscles while the contralateral leg served as an untrained within-subject control. The training protocol has been described previously $[19,20]$. Subjects performed four bouts of 125 isometric soleus contractions on five days per week. Computerized data logging equipment allowed subjects to perform the majority of training at home. Throughout the duration of the training protocol, subjects averaged 8270 soleus contractions per month.

Soleus physiologic adaptations to training were assessed on the date of H-reflex testing with the same apparatus and stimulation parameters used for training. With one exception (Subject 2) all Acute subjects likewise underwent one bout of repetitive soleus stimulation after H-reflex testing, using the same protocol administered to the Training cohort. Soleus 
torque for each contraction was expressed as a percent of the torque in contraction 1 (normalized torque). Fatigue index was calculated as the quotient of the final and first torque values, expressed as a percent. We calculated rate of torque rise and rate of torque fall between $20 \%$ and $80 \%$ of the peak force for each contraction, then normalized each rate to the peak torque of the corresponding contraction, as previously described [5].

The Acute cohort underwent H-reflex assessment within 7 weeks post-SCI (“AC"). The subjects in the Training cohort had an H-reflex assessment near 7 months post-SCI (mean time 0.62 years), at which point they had completed approximately 4 months of training (mean 0.3 years). These data comprise the "TR1" measurement. These subjects trained for up to three years (Table 1) and had another H-reflex assessment near the end of their training participation (mean time 2.5 years post-SCI, 2.2 years of training). The trained and untrained limbs were both assessed at this point, referred to as the "TR2" measurement. Three subjects in the Training cohort were initially tested in the Acute cohort; longitudinal data for these subjects are presented for comparison with our previous longitudinal report [17].

The tibial nerve was stimulated in the popliteal fossa with a double-pronged surfacestimulation electrode, placed so that the cathode was proximal to the anode. Two silversilver chloride electrodes recorded soleus compound muscle action potential activity. The maximal M-wave amplitude (Mmax) was determined via single-pulse stimulation. $\mathrm{H}$ reflexes were recruited on the ascending portion of the H-reflex recruitment curve [18] until stable H-reflexes of 20-40\% of Mmax were obtained [1,17]. Peak to peak amplitude for Mmax and H-reflexes were manually obtained (Datapac 2000, RunTechnologies, Laguna Hills, CA) and exported to spreadsheet software.

H-reflex depression was examined within fifteen-pulse stimulus trains elicited at various stimulation frequencies: $0.1 \mathrm{~Hz}, 0.2 \mathrm{~Hz}, 1 \mathrm{~Hz}, 2 \mathrm{~Hz}, 5 \mathrm{~Hz}$, and again at $0.1 \mathrm{~Hz}$. The second $0.1 \mathrm{~Hz}$ bout, denoted " $0.1 \mathrm{~s}$ ", was administered to verify that H-reflexes returned to their original amplitude after the stimulation bout, confirming that the stimulating electrode did not move during testing. Representative examples of H-reflex responses appear in Fig. 1. No H-reflex depression was expected to occur at the lowest frequency $(0.1 \mathrm{~Hz})$. In each $15-$ pulse train, the first (non-depressed) H-reflex response was excluded from analysis. For each subject, the mean amplitude of H-reflexes 2-15 at each frequency was then normalized to the mean value of the $0.1 \mathrm{~Hz}$ (non-depressing) condition.

For most subjects, H-reflexes recruited at the target amplitude (20-40\% of Mmax) were preceded by an M-wave. To further inspect for stimulator probe movement, we scrutinized the stability of the $\mathrm{M}$-waves preceding the $\mathrm{H}$ reflexes. We obtained the mean peak to peak amplitude of the M-waves preceding H-reflexes number $2-15$ at each frequency. We compared this value in the initial $0.1 \mathrm{~Hz}$ bout to the other frequencies to verify that tibial nerve activation differences did not underlie apparent $\mathrm{H}$-reflex depression at the higher frequencies. 
Finally, mean H-reflex amplitude at the initial and at the final $0.1 \mathrm{~Hz}$ conditions were also normalized to Mmax. This provides an index of the percent of the motor neuron pool that was recruited by tibial nerve stimulation at the beginning and at the end of the session.

We used a two-way (group $\times$ frequency) analysis of variance (ANOVA) to test for differences in H-reflex depression. We computed the coefficient of variation in H-reflex amplitude for each subject (pulses 2-15), then used a one-way repeated measures ANOVA to test for differences in variation between subject cohorts at each frequency condition. For each subject group, we used one-way repeated measures ANOVA to determine whether Mwaves preceding the H-reflexes varied according to frequency. Alpha for all tests was $p<$ 0.05. Because of limitations in statistical power, longitudinal H-reflex depression data for the three subjects who appeared in the both the Acute and the Training cohorts will be analyzed descriptively.

To characterize the training adaptations we analyzed torque, rate of torque rise, and rate of torque fall for each 25 th contraction in the 125-contraction bout. Each dependent variable was analyzed with a two-way (group $\times$ contraction number) ANOVA.

Mean (SD) H-reflex amplitude for the $0.1 \mathrm{~Hz}$ condition was $37.58 \%$ (19.81) of MaxM. This level of H-reflex recruitment did not differ $(p>0.05)$ at the second $0.1 \mathrm{~Hz}$ condition $(0.1 \mathrm{~s})$, supporting the uniformity of test conditions at the beginning and end of the experiment. $\mathrm{H}$ reflex amplitude at $1 \mathrm{~Hz}, 2 \mathrm{~Hz}$, and $5 \mathrm{~Hz}$ was significantly lower than at $0.1 \mathrm{~Hz}, 0.2 \mathrm{~Hz}$, and $0.1 \mathrm{~s}$ (all $p<0.05$ ) (Fig. 2), indicating that H-reflex depression occurred at the highest frequencies. The pattern of depression differed significantly between the Acute (AC) and Untrained (UN) cohorts $(p<0.05$ ). Mean (SD) Hreflex amplitude (normalized to $0.1 \mathrm{~Hz}$ ) for AC was $92.83 \%$ (10.72\%), 39.99\% (28.09\%), 24.56\% (17.13\%), and $13.39 \%$ (7.10\%) for $0.2 \mathrm{~Hz}, 1 \mathrm{~Hz}, 2 \mathrm{~Hz}$, and $5 \mathrm{~Hz}$ respectively (Fig. 2). Comparable values for UN were 99.73\% (42.39\%), 66.16\% (54.27\%), 71.93\% (75.23\%), and 60.71\% (43.18\%). H-reflex amplitude for the two Training conditions (TR1 and TR2) was intermediate between AC and UN ( $p>$ $0.05)$ and did not differ from one another $(p>0.05)$. No significant differences existed between TR2 and UN coefficients of variation, suggesting that training did not affect the homogeneity of H-reflex responses during repetitive stimulation.

Mean (SD) normalized amplitude of $\mathrm{M}$-waves preceding the $\mathrm{H}$ reflexes was $23.92 \%$ (20.28) for AC, $15.64 \%$ (14.20) for TR1, and $13.57 \%$ (10.84) for TR2. M-wave amplitude did not differ according to frequency for any of these conditions $(p>0.05)$; thus the H-reflex depression observed at the higher frequencies was not due to reduced recruitment of the tibial nerve alpha motor neuronal pool. For the UN cohort, mean (SD) normalized M-wave amplitude was $12.62 \%$ (11.51). M-waves for $5 \mathrm{~Hz}$ were significantly higher than for $0.1 \mathrm{~Hz}$ $(p<0.05)$.

Soleus torque was lowest for TR1 (33.6 N m) and highest for TR2 (47.7 N m). Training led to partial preservation of soleus torque over the 2 years studied (Fig. 3A). Training attenuated but did not eliminate the normally occurring decline in normalized torque in paralyzed soleus muscle. 
Mean FI for the trained limbs at year 1 and year 2 was significantly higher than the untrained limbs $(p<0.05)$ (Fig. 3A). While FI at year 1 was no different from Acute FI $(p>$ $0.05)$, FI at year 2 was significantly lower than Acute FI $(p<0.05)$. Thus training attenuated but did not eliminate the normally occurring loss of fatigue resistance in paralyzed soleus muscle.

For normalized rate of torque rise, a significant interaction existed between contraction number and subject group (Fig. 3B) $(p<0.05)$. At contraction 1, rate of rise for UN was significantly higher than for the other cohorts $(p<0.05)$. By contraction 25 , UN continued to differ only from TR2. As fatigue developed during the repetitive stimulation (Fig. 3A), contractile slowing emerged most notably in UN and TR2 (Fig. 3B). By contraction 100, rate of rise for these groups was significantly lower than for either AC or TR1. Thus 2 years of training had mixed results on the rate of torque rise. While trained muscles did not demonstrate elevated rate of torque rise at the start of stimulation (unlike UN), the trained muscles experienced fatigue-related contractile slowing during repetitive stimulation (like $\mathrm{UN})$.

Likewise, for the normalized rate of force fall, a significant interaction existed between contraction number and subject group (Fig. 3C) $(p<0.05)$. At contraction 1, rate of fall for $\mathrm{UN}$ was significantly higher than for the other cohorts $(p<0.05)$. However, by contraction 25 the rate of fall for AC exceeded the rate for TR2, and from contraction 50 onward the AC rate also exceeded UN. Thus as was seen above for torque rise, 2 years of training also had mixed results on the rate of torque fall. While trained muscles did not demonstrate elevated rate of torque fall at the start of stimulation (unlike UN), the trained muscles experienced fatigue-related contractile slowing during repetitive stimulation (like UN).

Data from the three longitudinal training subjects appear in Fig. 4. Trained limb depression was intermediate between the Acute and Untrained conditions. No depression occurred in the untrained limbs at $1 \mathrm{~Hz}$ or $2 \mathrm{~Hz}$. In contrast, $\mathrm{H}$-reflexes were depressed by $\sim 50 \%$ at 1 and $2 \mathrm{~Hz}$ in the trained limbs at TR2.

Subjects with complete SCI demonstrated noteworthy adaptations of soleus torque, FI, and contractile speed after 2 years of training. Contrary to our hypothesis, these physiologic adaptations were not conclusively associated with normalization of H-reflex depression. Though trained limb depression values were intermediate between Acute and Untrained values, between-limb differences did not reach statistical significance. However, three subjects who began training very early after SCI demonstrated partial reversal of depression loss at the 2nd year of measurement. More work is required to determine whether muscle stimulation exerts a normalizing influence on spinal circuitry.

In a subject we previously followed longitudinally over 44 weeks, strong negative correlations existed between fatigue resistance and H-reflex depression [17]. Given recent findings regarding the retrograde transport of neurotrophic factors from exercising muscle [6,7], we hypothesized that reversal of physiologic decline may be accompanied by restoration of H-reflex depression. This hypothesis was supported for the subjects in the longitudinal sub-cohort who began training very quickly post-injury (Fig. 4). However, 
restoration of H-reflex depression was equivocal in the remainder of the Training cohort. The apparent dissociation of physiologic and reflex parameters in this cohort may suggest that electrical stimulation is inferior to other modes of exercise (e.g. cycling) as a neuromodulatory influence. Alternatively, between-limb differences in H-reflex depression could have been undermined if cross-education existed between the trained and untrained limbs. However, cross-education of H-reflexes was not observed in a previous unilateral plantar flexion training protocol [12] and is likely to depend heavily on supra-spinal mechanisms [3].

Short-term changes in H-reflex depression have been observed in humans with incomplete SCI who performed a single bout of voluntary-assisted cycling [15]. Two case reports suggest that passive cycling [9] and treadmill walking [22] tend to foster a return of H-reflex depression in individuals with incomplete SCI. The present study is the first to explore the effects of training on H-reflex depression in individuals with complete SCI, and it is the first to examine a mode of exercise that does not involve rhythmic cyclical activity (cycling, locomotion). Because animal models suggest that activity in proprioceptive pathways may be a key trigger for H-reflex normalization [14], isometric muscle stimulation might seem less than ideal. Thus, the degree of normalization of H-reflex depression observed with the current isometric training protocol is a novel finding. Perhaps routine muscle activation in the absence of joint movement offers an alternative mode of afferent input to trigger normalization of the H-reflex after SCI. Conversely, perhaps the routine muscle contractions triggered retrograde axonal signaling mechanisms (e.g. brain-derived neurotrophic factor, neurotrophin-3) [7] that fostered spinal plasticity. The close association between spasticity and the loss of post activation depression underscores the importance of interventions that affect this pathway [8]. Although the mechanism is not clear, these findings suggest that neural plasticity of the spinal cord, at least with respect to this pathway, is modulated by chronic repetitive isometric muscle training in humans with SCI.

Physiologic adaptations after 2 years of training were associated with partial normalization of H-reflex depression. Results from three subjects who began training very early after SCI suggest that timing of training may be important. More work is required to determine whether muscle stimulation exerts a normalizing influence on spinal circuitry after SCI.

\section{Acknowledgments}

This work was supported by awards from the NIH (R01NR010285; R01HD062507) and the Craig H. Neilsen Foundation.

\section{Abbreviations}

$\begin{array}{ll}\text { SCI } & \text { spinal cord injury } \\ \text { AC } & \text { acute } \\ \text { UN } & \text { untrained } \\ \text { TR1 } & \text { training year 1 } \\ \text { TR2 } & \text { training year 2. }\end{array}$




\section{References}

1. Aagaard P, Simonsen EB, Andersen JL, Magnusson P, Dyhre-Poulsen P. Neural adaptation to resistance training: changes in evoked V-wave and H-reflex responses. J. Appl. Physiol. 2002; 92:2309-2318. [PubMed: 12015341]

2. American Spinal Injury Association. International Standards for Neurological Classification of SCI. Atlanta, Georgia: American Spinal Injury Association; 2002.

3. Carroll TJ, Herbert RD, Munn J, Lee M, Gandevia SC. Contralateral effects of unilateral strength training: evidence and possible mechanisms. J. Appl. Physiol. 2006; 101:1514-1522. [PubMed: 17043329]

4. DiStefano PS, Friedman B, Radziejewski C, Alexander C, Boland P, Schick CM, Lindsay RM, Wiegand SJ. The neurotrophins BDNF, NT-3, and NGF display distinct patterns of retrograde axonal transport in peripheral and central neurons. Neuron. 1992; 8:983-993. [PubMed: 1375039]

5. Dudley-Javoroski S, Littmann AE, Iguchi M, Shields RK. Doublet stimulation protocol to minimize musculoskeletal stress during paralyzed quadriceps muscle testing. J. Appl. Physiol. 2008; 104:1574-1582. [PubMed: 18436697]

6. Dupont-Versteegden EE, Houle JD, Dennis RA, Zhang J, Knox M, Wagoner G, Peterson CA. Exercise-induced gene expression in soleus muscle is dependent on time after spinal cord injury in rats. Muscle Nerve. 2004; 29:73-81. [PubMed: 14694501]

7. Gomez-Pinilla F, Ying Z, Opazo P, Roy RR, Edgerton VR. Differential regulation by exercise of BDNF and NT-3 in rat spinal cord and skeletal muscle. Eur. J. Neurosci. 2001; 13:1078-1084. [PubMed: 11285004]

8. Grey MJ, Klinge K, Crone C, Lorentzen J, Biering-Sorensen F, Ravnborg M, Nielsen JB. Postactivation depression of soleus stretch reflexes in healthy and spastic humans. Exp. Brain Res. 2008; 185:189-197. [PubMed: 17932663]

9. Kiser TS, Reese NB, Maresh T, Hearn S, Yates C, Skinner RD, Pait TG, Garcia-Rill E. Use of a motorized bicycle exercise trainer to normalize frequency-dependent habituation of the H-reflex in spinal cord injury. J. Spinal Cord Med. 2005; 2:241-245. [PubMed: 16048142]

10. Knikou M, Angeli CA, Ferreira CK, Harkema SJ. Soleus H-reflex modulation during body weight support treadmill walking in spinal cord intact and injured subjects. Exp. Brain Res. 2009; 193:397-407. [PubMed: 19011843]

11. Knikou M, Conway BA. Reflex effects of induced muscle contraction in normal and spinal cord injured subjects. Muscle Nerve. 2002; 26:374-382. [PubMed: 12210367]

12. Lagerquist O, Zehr EP, Docherty D. Increased spinal reflex excitability is not associated with neural plasticity underlying the cross-education effect. J. Appl. Physiol. 2006; 100:83-90. [PubMed: 16357081]

13. Munson JB, Foehring RC, Mendell LM, Gordon T. Fast-to-slow conversion following chronic low-frequency activation of medial gastrocnemius muscle in cats. II. Motoneuron properties. J. Neurophysiol. 1997; 77:2605-2615. [PubMed: 9163379]

14. Ollivier-Lanvin K, Keeler BE, Siegfried R, Houle JD, Lemay MA. Proprioceptive neuropathy affects normalization of the H-reflex by exercise after spinal cord injury. Exp. Neurol. 2010; 221:198-205. [PubMed: 19913536]

15. Phadke CP, Flynn SM, Thompson FJ, Behrman AL, Trimble MH, Kukulka CG. Comparison of single bout effects of bicycle training versus locomotor training on paired reflex depression of the soleus H-reflex after motor incomplete spinal cord injury. Arch. Phys. Med. Rehabil. 2009; 90:1218-1228. [PubMed: 19577036]

16. Reese NB, Skinner RD, Mitchell D, Yates C, Barnes CN, Kiser TS, Garcia-Rill E. Restoration of frequency-dependent depression of the H-reflex by passive exercise in spinal rats. Spinal Cord. 2006; 44:28-34. [PubMed: 16044168]

17. Schindler-Ivens S, Shields RK. Low frequency depression of H-reflexes in humans with acute and chronic spinal-cord injury. Exp. Brain Res. 2000; 133:233-241. [PubMed: 10968224]

18. Schindler-Ivens SM, Shields RK. Comparison of linear regression and probit analysis for detecting H-reflex threshold in individuals with and without spinal cord injury. Electromyogr. Clin. Neurophysiol. 2004; 44:153-159. [PubMed: 15125055] 
19. Shields RK, Dudley-Javoroski S. Musculoskeletal adaptation in chronic spinal cord injury: effects of long-term soleus electrical stimulation training. J. Neurorehabil. Neural Repair. 2006; 21:169179.

20. Shields RK, Dudley-Javoroski S. Musculoskeletal plasticity after acute spinal cord injury: effects of long-term neuromuscular electrical stimulation training. J. Neurophysiol. 2006; 95:2380-2390. [PubMed: 16407424]

21. Skinner RD, Houle JD, Reese NB, Berry CL, Garcia-Rill E. Effects of exercise and fetal spinal cord implants on the H-reflex in chronically spinalized adult rats. Brain Res. 1996; 729:127-131. [PubMed: 8874885]

22. Trimble MH, Kukulka CG, Behrman AL. The effect of treadmill gait training on low-frequency depression of the soleus H-reflex: comparison of a spinal cord injured man to normal subjects. Neurosci. Lett. 1998; 246:186-188. [PubMed: 9792623] 

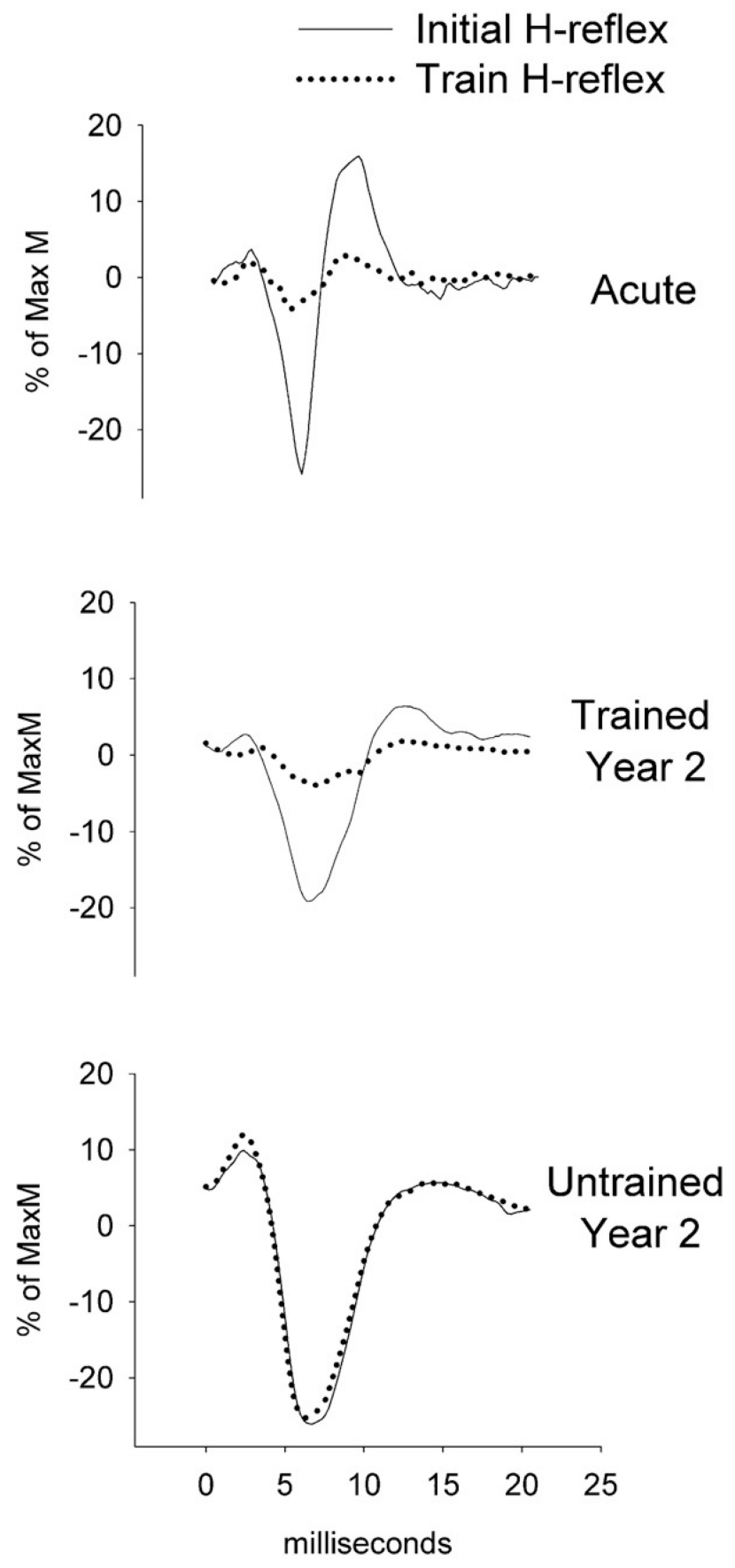

Fig. 1.

Representative example of H-reflex suppression in a single subject (Subject 6, Table 1) at AC, TR2 and UN. Data are from the initial (non-depressed) and subsequent (depressed) Hreflex response in a $1 \mathrm{~Hz}$ stimulation train. 


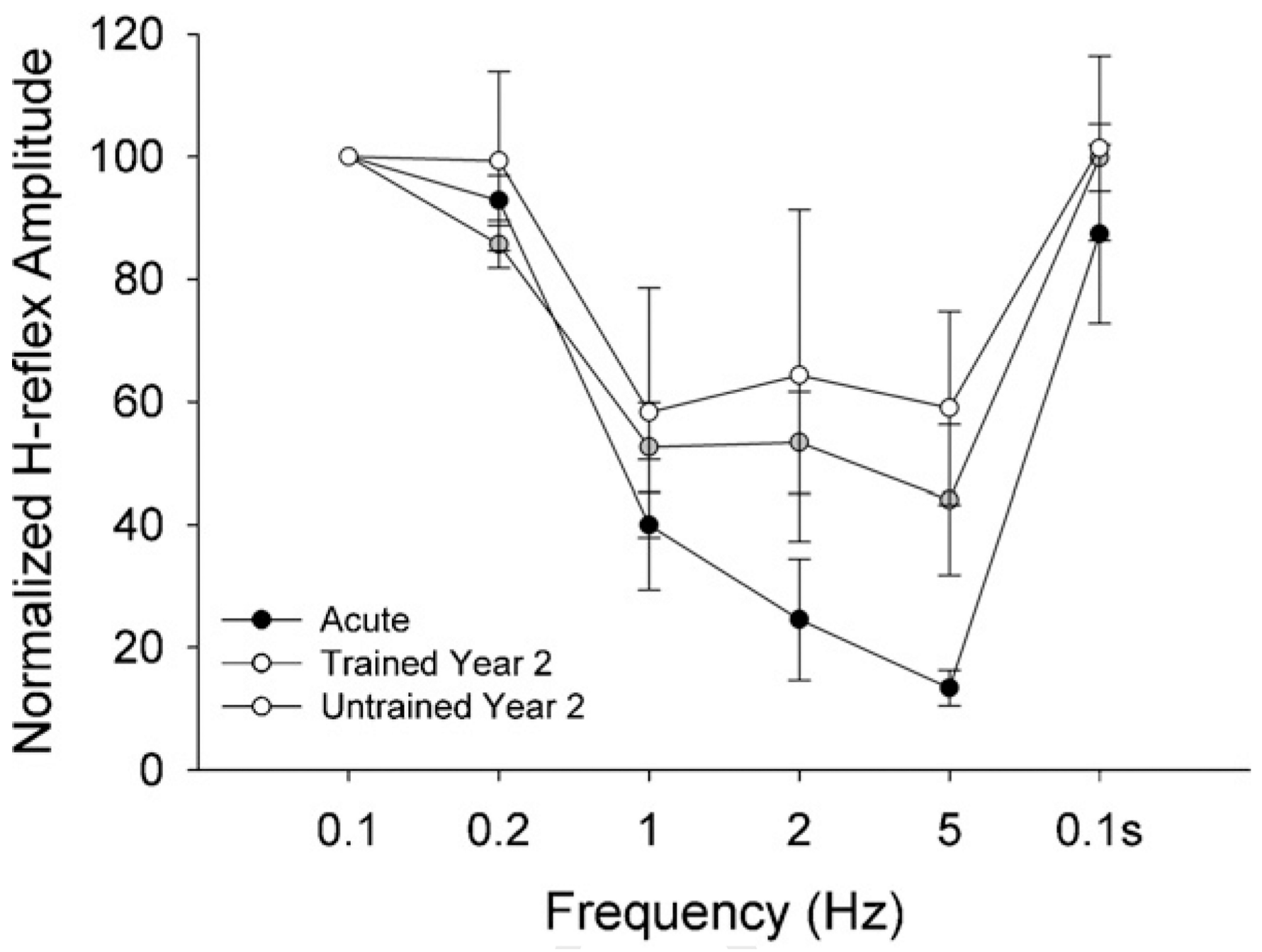

Fig. 2.

H-reflex suppression for four subject cohorts at several stimulation frequencies. Values are mean (SE) of pulses 2-15 at each frequency, normalized to the mean of the $0.1 \mathrm{~Hz}$ (nonsuppressing) condition. Among the subject cohorts, the pattern of suppression for the Acute group differed significantly from the Untrained group $(p<0.05)$. Overall suppression at 1 $\mathrm{Hz}, 2 \mathrm{~Hz}$ and $5 \mathrm{~Hz}$ was significantly lower than $0.1 \mathrm{~Hz}, 0.2 \mathrm{~Hz}$, and $0.1 \mathrm{~s}(p<0.05)$. 
A
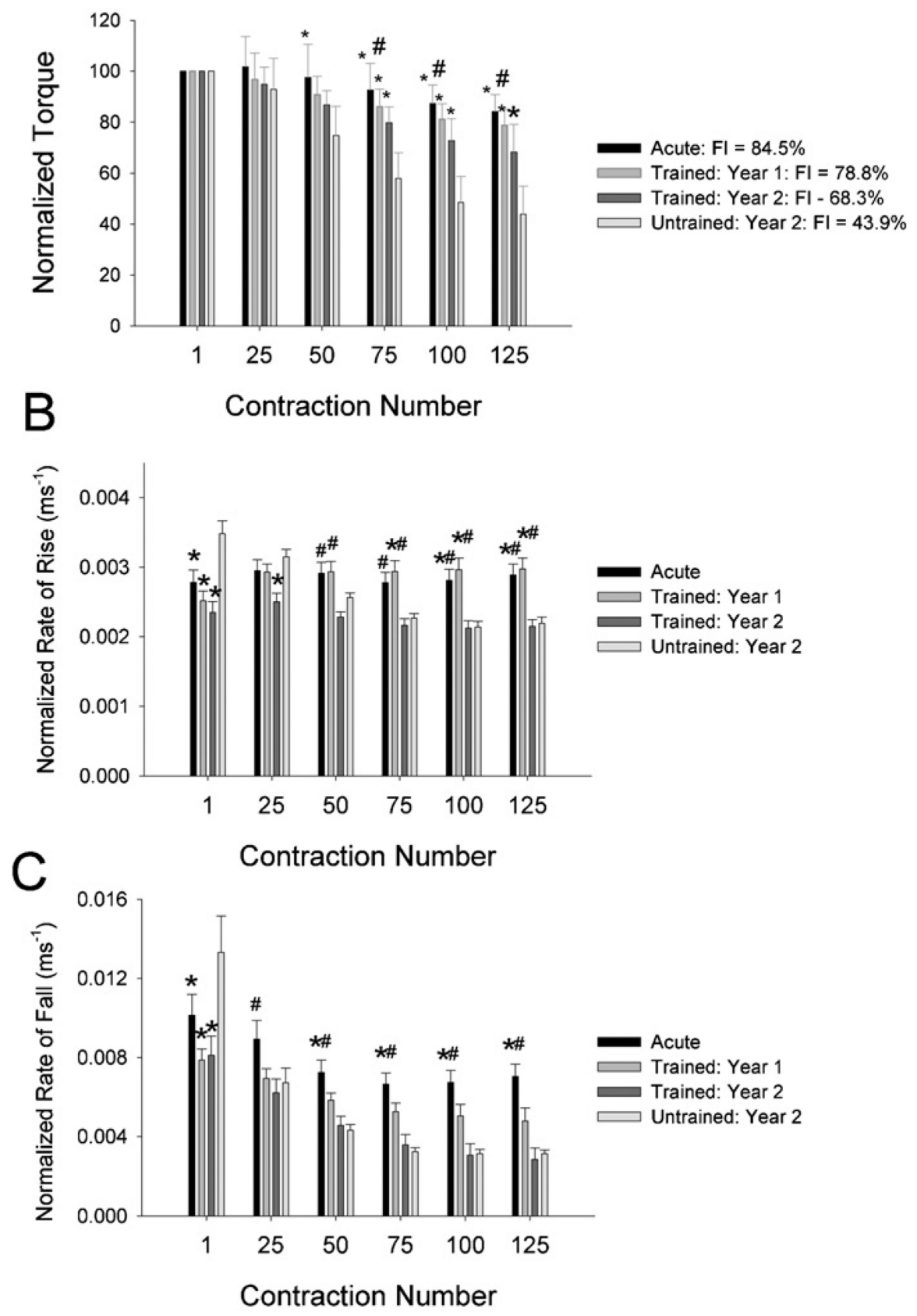

Fig. 3.

Training effects for soleus physiology parameters. Values are mean (SE). FI = fatigue index. *Significantly different from the Untrained group $(p<0.05)$. ${ }^{\#}$ Significantly different from the Trained: year 2 group $(p<0.05)$. 


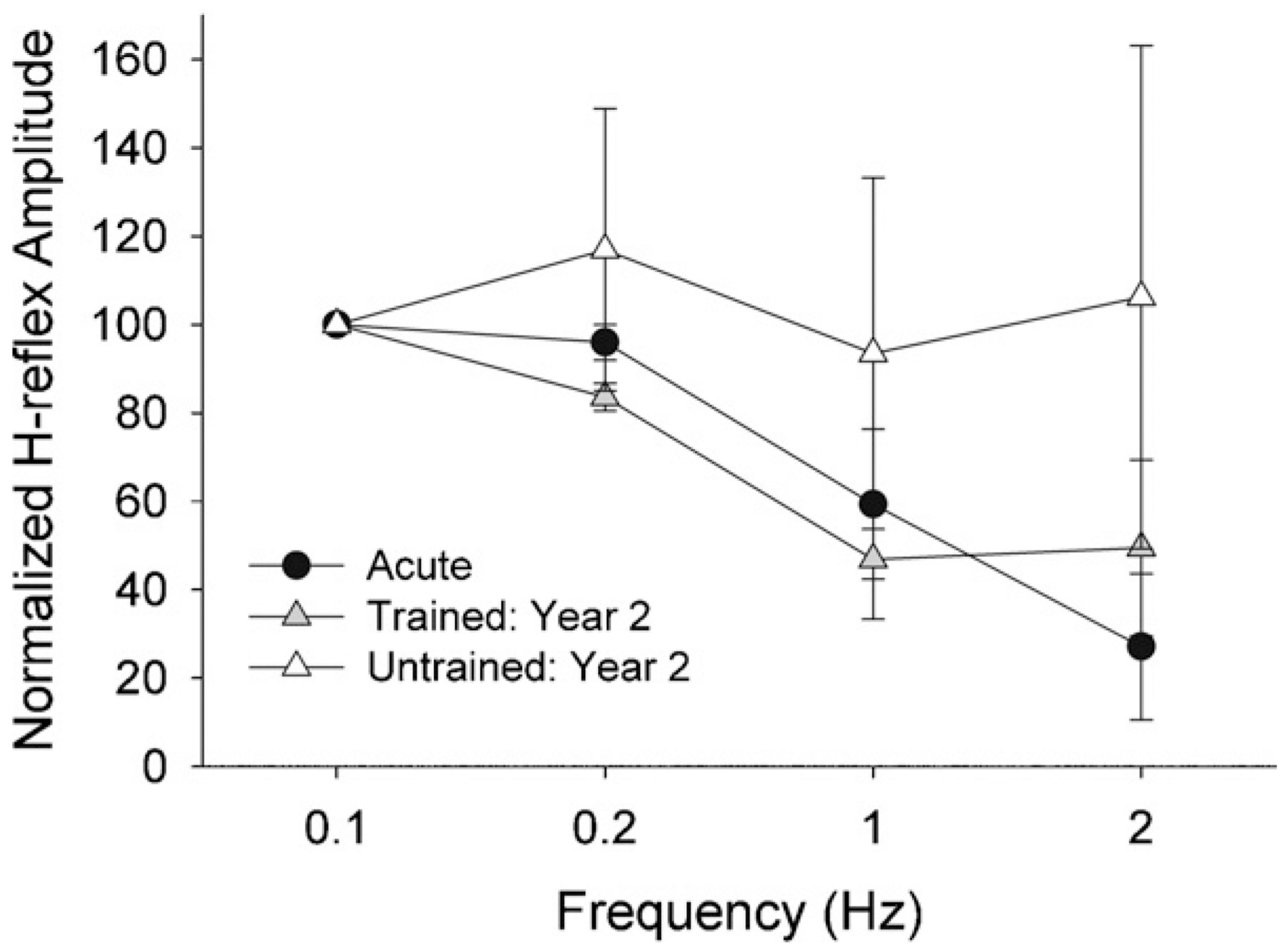

Fig. 4.

H-reflex suppression for subjects followed longitudinally through the Acute and Training cohorts. Mean (SE) H-reflex amplitude for pulses 2-15 at each frequency, normalized to the mean of the $0.1 \mathrm{~Hz}$ (non-suppressing) condition. 
